04

\title{
Проявление рассеяния излучения в методе импульсной терагерцовой спектроскопии
}

\author{
() В.Л. Малевич ${ }^{1}$, Г.В. Синицын ${ }^{1}$, Г.Б. Сочилин ${ }^{2}$, Н.Н. Розанов ${ }^{2,3,4}$ \\ ${ }^{1}$ Институт фозики НАН Беларуси, \\ 220072 Минск, Беларусь \\ ${ }^{2}$ Государственный оптический институт им. С.И. Вавилова, \\ 199053 Санкт-Петербург, Россия \\ ${ }^{3}$ Университет ИТМО, \\ 197101 Санкт-Петербург, Россия \\ ${ }^{4}$ Физико-технический институт им. А.Ф. Иофффе, \\ 194021 Санкт-Петербург, Россия \\ e-mail: v.malevich@ifanbel.bas-net.by, nnrosanov@mail.ru
}

Поступила в редакцию 12.02.2018 г.

\begin{abstract}
Проведен анализ рассеяния широкополосных терагерцовых импульсов шероховатой поверхностью полубесконечной среды модифицированным методом Монте-Карло, учитывающим фазу излучения. Результаты сравнены с упрощенным аналитическим выражением, которое допускает обобщение на случай слоистых сред и наличия объемного рассеяния. Соответствие этих результатов позволяет предложить простой способ исключения или ослабления влияния рассеяния на восстановление характеристик сред в методе импульсной терагерцовой спектроскопии.
\end{abstract}

DOI: $10.21883 /$ OS.2018.06.46090.40-18

\section{Введение}

Метод импульсной терагерцовой (ТГц) спектроскопии с временным разрешением основан на измерении временной зависимости электрического поля широкополосного ТГц импульса, прошедшего через исследуемый объект или отраженного от него $[1,2]$. Сравнивая спектр этого импульса с опорным спектром известного образца, можно получить спектральные зависимости показателя преломления и коэффициента поглощения исследуемого образца в широкой области частот, простирающейся от СВЧ до дальнего ИК диапазона. В импульсной ТГц спектроскопии используется информация и о фазе ТГц импульса. Поэтому результаты спектральных измерений могут оказаться весьма чувствительными к эффекту рассеяния на случайных неоднородностях образца [3-10], который приводит к искажениям волнового фронта и временной формы ТГц импульса.

Большинство материалов сильно поглощают ТГц излучение, и поэтому для измерения их спектров используется метод импульсной ТГц спектроскопии на отражение. В этом случае можно ожидать, что главную роль будет играть рассеяние ТГц импульса на шероховатой поверхности образца $[3,4]$. В настоящей работе численно моделируется пространственно-временная эволюция электрического поля ТГц импульса с гауссовым поперечным профилем, отражающегося от шероховатой поверхности среды с лоренцевым контуром поглощения, и исследуется влияние шероховатости на спектр отражения. Соответствие численных результатов и аналитических выводов упрощенного подхода позволяет предложить простой вариант значительного ослабления влияния рассеяния излучения на неоднородностях на точность восстановления характеристик сред методом ТГц импульсной спектроскопии.

\section{Монте-Карло-моделирование}

В отличие от работы [10], в которой случайным образом разыгрывалась поверхность раздела сред, и далее рассчитывалось регулярное распространение пучка-импульса, здесь будем проводить моделирование с использованием модифицированного метода МонтеКарло $[11,12]$, учитывающего фазу электрического поля излучения и когерентные эффекты. В данном методе моделируется эволюция электрического поля излучения, которое представляется в виде ансамбля плоских волн, каждая из которых характеризуется частотой, волновым вектором, амплитудой, поляризацией и фазой. Такой метод был успешно применен для моделирования многократного рассеяния поляризованного света в случайнонеоднородной среде [11], эффекта когерентного обратного рассеяния [13], а также распределения амплитуды и фазы света при его острой фокусировке в рассеивающей среде [14]. Принципиально при достаточно большой статистике данный подход эквивалентен использованному в [10], но он легче обобщается на случаи слоистых сред и объемного рассеяния.

Рассматриваем отражение ТГц импульса от шероховатой поверхности полубесконечной среды с диэлектрической проницаемостью, зависящей от частоты (некоторые обобщения обсуждаются в заключении). Для простоты предположим, что в срединной плоскости $(z=0)$ рассе- 
ивающей поверхности волновой фронт падающего вдоль оси $z$ импульса является плоским. Предполагаем также, что падающий ТГц импульс характеризуется гауссовым поперечным распределением амплитуды, а его временная форма имеет биполярный вид. Пространственновременное распределение поля такого импульса будем аппроксимировать выражением

$$
\begin{gathered}
E_{i}(t, z=0, \rho)=A(t) \exp \left(-\rho^{2} / w^{2}\right), \\
A(t)=a t^{2}(1-t / 3 \tau) \exp (-t / \tau), \quad t>0,
\end{gathered}
$$

где параметры $w, \tau$ и $a$ характеризуют радиус пучка, длительность и амплитуду импульса соответственно.

Профиль шероховатой поверхности $z=\zeta(x, y)(\xi-$ высота неоднородности относительно плоскости $z=0$ ) будем описывать гауссовой статистикой с корреляционной функцией $\left\langle\xi(\boldsymbol{\rho}) \xi\left(\boldsymbol{\rho}^{\prime}\right)\right\rangle=\sigma^{2} \exp \left[-\left(\boldsymbol{\rho}-\boldsymbol{\rho}^{\prime}\right)^{2} / 2 l^{2}\right]$, где $\boldsymbol{\rho}=(x, y)$ - двумерный радиус-вектор в срединной плоскости, $\sigma$ и $l$ - среднеквадратичная высота и радиус корреляции (поперечный размер) неоднородностей. Рассеяние плоских волн будем рассматривать в приближении Кирхгофа $[15,16]$, которое справедливо для крупномасштабных и плавных неоднородностей с радиусом кривизны, значительно превышающим длину волны излучения. В этом случае рассеивающую поверхность можно считать локально-плоской и для коэффициентов отражения и преломления использовать формулы Френеля, параметрами которых являются локальный угол падения плоской волны и показатели преломления граничащих сред.

Алгоритм Монте-Карло-моделирования организован следующим образом. Из выражения (1) путем фурьепреобразования вычисляем угловой и частотный спектры электрического поля ТГц импульса:

$$
\begin{aligned}
E\left(\omega, \mathbf{k}_{t}\right) & =\int_{0}^{+\infty} d t \int d^{2} \boldsymbol{\rho} A(t) \exp \left(-\rho^{2} / \omega^{2}\right) e^{-i\left(\omega t-\mathbf{k}_{\boldsymbol{f}} \boldsymbol{\rho}\right)} \\
& =\frac{2 \pi a \omega^{2}|\omega|}{\left(\omega^{2}+\tau^{-2}\right)} e^{i \varphi(\omega)} e^{-\frac{k_{t}^{2} \omega^{2}}{4}} \\
& \varphi(\omega)=\frac{\pi}{2} \operatorname{sgn}(\omega)+\operatorname{arctg}\left(\frac{1}{\omega \tau}\right) .
\end{aligned}
$$

Далее методом статистических испытаний из выражения (2) находится начальное распределение плоских волн по частотам $\omega$, фазам $\varphi(\omega)$ и поперечным волновым векторам $\mathbf{k}_{t}$. Компонента $k_{z}$ волнового вектора вдоль оси $z$ находится из соотношений $k_{t}^{2}+k_{z}^{2}=\omega^{2} / c^{2}$, где $k_{t} \leq \omega / c(c-$ скорость света в вакууме $)$. Выполнение этого неравенства означает, что мы пренебрегаем эванесцентными волнами, которые экспоненциально затухают в пространстве и не дают вклада в дальнее поле. Вектор поляризации отдельной плоской волны определяется из условия поперечности. Амплитуда, поляризация и направление волнового вектора плоской волны, отраженной шероховатой поверхностью, находятся из формул Френеля. Единичный вектор внешней нормали $\mathbf{n}$ к поверхности, который определяет угол падения плоской волны, находится [16] из выражений

$$
\mathbf{n}=\frac{-\hat{\mathbf{z}}+\gamma_{2, x} \hat{\mathbf{x}}+\gamma_{2, y} \hat{\mathbf{y}}}{\sqrt{1+\gamma_{2, x}^{2}}+\gamma_{2, y}^{2}}, \quad \gamma_{2}=\frac{\partial \xi}{\partial x} \hat{\mathbf{x}}+\frac{\partial \xi}{\partial y} \hat{\mathbf{y}},
$$

где $\hat{\mathbf{x}}, \hat{\mathbf{y}}$ и $\hat{\mathbf{z}}-$ орты координат, двумерный вектор $\gamma_{2}$ характеризует наклон поверхности. Для изотропной поверхности, подчиняющейся гауссовой статистике, функция распределения $\xi(\gamma)$, описывающая наклон поверхности, также является гауссовой и имеет вид $\xi\left(\gamma_{2}\right)=\left(1 / 2 \pi \overline{\gamma_{2}^{2}}\right) e^{-\gamma_{2}^{2} / 2 \overline{\gamma_{2}^{2}}}$, где $\overline{\gamma_{2}^{2}}=\sigma^{2} / l^{2}$. Компоненты вектора $\gamma_{2}$ находим из соотношений

$$
\gamma_{2}=-\sqrt{2 \overline{\gamma_{2}^{2}}} \ln q, \quad \gamma_{2, x}=\gamma_{2} \cos 2 \pi q_{1}, \quad \gamma_{2, y}=\gamma_{2} \sin 2 \pi q_{1},
$$

где $q$ и $q_{1}$ - случайные числа, равномерно распределенные в интервале $(0,1)$. Высота неоднородностей определяется из выражения $\xi=\sigma\left(\sum_{i=1}^{M} q_{i}-M / 2\right)\left(q_{i}-\right.$ однородно распределенные в интервале $(0,1)$ случайные числа), которое при $M \geq 6$ достаточно точно аппроксимирует нормальное распределение. Единичный вектор $\mathbf{r}$ вдоль направления отражения волны определяется как $\mathbf{r}=2 \cos \theta_{i} \mathbf{n}+\mathbf{i}$, где $\theta_{i}-$ угол падения падающей волны, определяемый соотношением $\cos \theta_{i}=|(\mathbf{n i})|, \mathbf{i}-$ единичный вектор вдоль направления падения волны.

Представим электрическое поле $\mathbf{E}_{i}$ падающей волны в виде

$$
\begin{gathered}
\mathbf{E}_{i}=\mathbf{E}_{i s}+\mathbf{E}_{i p}, \mathbf{E}_{i s}=\frac{\left(\mathbf{E}_{i}[\mathbf{n} \times \mathbf{i}]\right)[\mathbf{n} \times \mathbf{i}]}{([\mathbf{n} \times \mathbf{i}][\mathbf{n} \times \mathbf{i}])}, \\
\mathbf{E}_{i p}=\frac{\left(\mathbf{E}_{i}[\mathbf{i}[\mathbf{n i}]]\right)[\mathbf{i}[\mathbf{n i}]]}{([\mathbf{n} \times \mathbf{i}][\mathbf{n} \times \mathbf{i}])} .
\end{gathered}
$$

Здесь $\mathbf{E}_{i s}$ и $\mathbf{E}_{i p}$ соответствуют $s$ - и $p$-поляризованным компонентам электрического поля. Умножая компоненты падающего поля на коэффициенты френелевского отражения $r_{p}$ и $r_{s}$ для двух поляризаций, получаем из (4) для поля отраженной волны $E_{r}$ следующее выражение:

$$
\mathbf{E}_{r}=r_{s} \mathbf{E}_{i s}+r_{p} \mathbf{E}_{i p} .
$$

Пространственно-временное распределение когерентного электрического поля восстанавливается из углового и частотного спектров рассеянных волн с использованием обратного преобразования Фурье. При этом учитывается изменение фазы волны при ее распространении в свободном пространстве, а также в результате отражения от шероховатой поверхности.

\section{Результаты расчетов}

С целью проверки корректности развитого в настоящей работе подхода нами было проведено моделирование рассеяния широкополосного ТГц импульса шероховатой поверхностью $(\sigma=0.001 \mathrm{~cm}, l=0.2 \mathrm{~cm})$ среды 


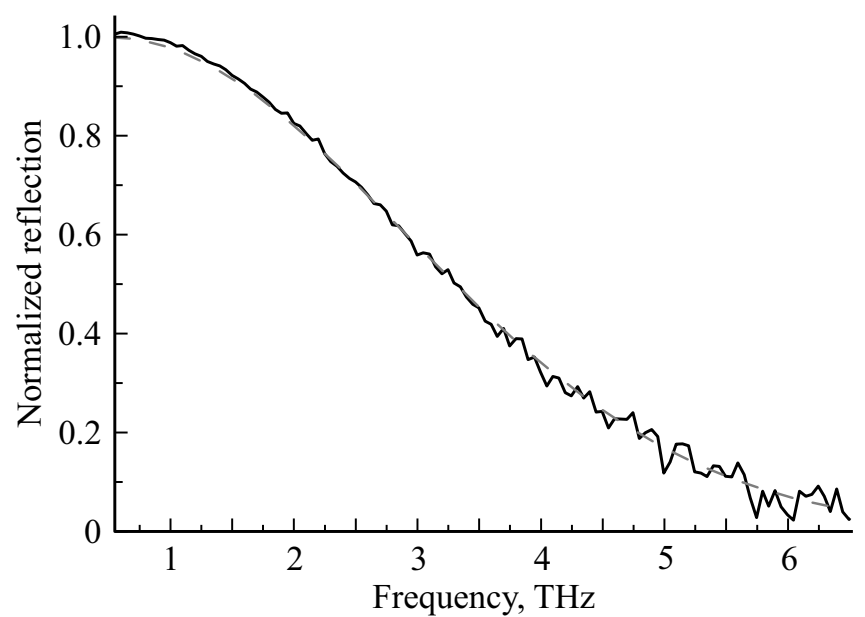

Рис. 1. Спектральная зависимость нормированного коэффициента отражения от шероховатой поверхности с параметрами $\sigma=0.001 \mathrm{~cm}, l=0.2 \mathrm{~cm}$, рассчитанная методом Монте-Карло (сплошная кривая). Штриховая кривая - результат аналитического расчета.

в отсутствие дисперсии и рассчитана когерентная компонента электрического поля отраженного излучения в дальней зоне. Чтобы исключить влияние дифракции, поперечное распределение амплитуды поля падающей волны предполагалось однородным. На рис. 1 приведена рассчитанная частотная зависимость эффективного коэффициента отражения в зеркальном направлении, нормированного на коэффициент отражения от гладкой поверхности. На этом же рисунке показана частотная зависимость фактора $\exp \left(2 \sigma^{2} \omega^{2} / c^{2}\right)[15,16]$, описывающего экспоненциальное уменьшение когерентного коэффициента отражения с увеличением частоты и характерной высоты неровности поверхности. Совпадение результатов Монте-Карло-моделирования с аналитическим решением в достаточно широкой спектральной области $[15,16]$ подтверждает корректность разработанных алгоритма и программы.

Также нами была рассчитана пространственновременная эволюция биполярного ТГц импульса с гауссовым поперечным профилем (1), распространяющегося в свободном пространстве, и проведено сравнение результатов моделирования с известным решением, справедливым в параксиальном приближении $[1,17]$ :

$$
E(t, \rho, z)=\frac{1}{2 \pi z c} \int d^{2} \boldsymbol{\rho}^{\prime} e^{-\frac{\left(\boldsymbol{\rho}-\boldsymbol{\rho}^{\prime}\right)^{2}}{w^{2}}} \frac{\partial A\left(t-z / c-\rho^{\prime 2} / 2 z c\right)}{\partial t}
$$

В качестве параметров ТГц импульса в начальной плоскости $z=0$ были выбраны $w=0.5 \mathrm{~cm}, \tau=0.2 \mathrm{ps}$. Из результатов моделирования следует (рис. 2,a), что ограниченность ТГц импульса в поперечном сечении приводит к изменению его пространственно-временной структуры по мере распространения. Данный эффект проявляется в наибольшей степени для широкополосных импульсов, состоящих из нескольких осцилляций поля, и обусловлен зависимостью дифракционной длины от частоты излучения [18]. В результате дифракции первоначально однородное по сечению пучка распределение частотных компонент поля становится неоднородным. Низкочастотные компоненты поля дифрагируют быстрее, чем высокочастотные, и смещаются на периферию пучка. В результате временной профиль ТГц импульса сужается на оси пучка и расширяется на его периферии. Этот эффект иллюстрирует рис. 2, $b$, где приведены результаты расчетов временных профилей ТГц импульса для двух значений $\rho$. Результаты расчетов методом Монте-Карло и с использованием выражения (6) хорошо совпадают, что указывает на корректность используемых алгоритма и программы моделирования.

Применим модифицированный метод Монте-Карло для моделирования рассеяния биполярного ТГц импульса с гауссовым поперечным профилем, падающего нормально на шероховатую поверхность полубесконечной среды, характеризующейся диэлектрической проницае-
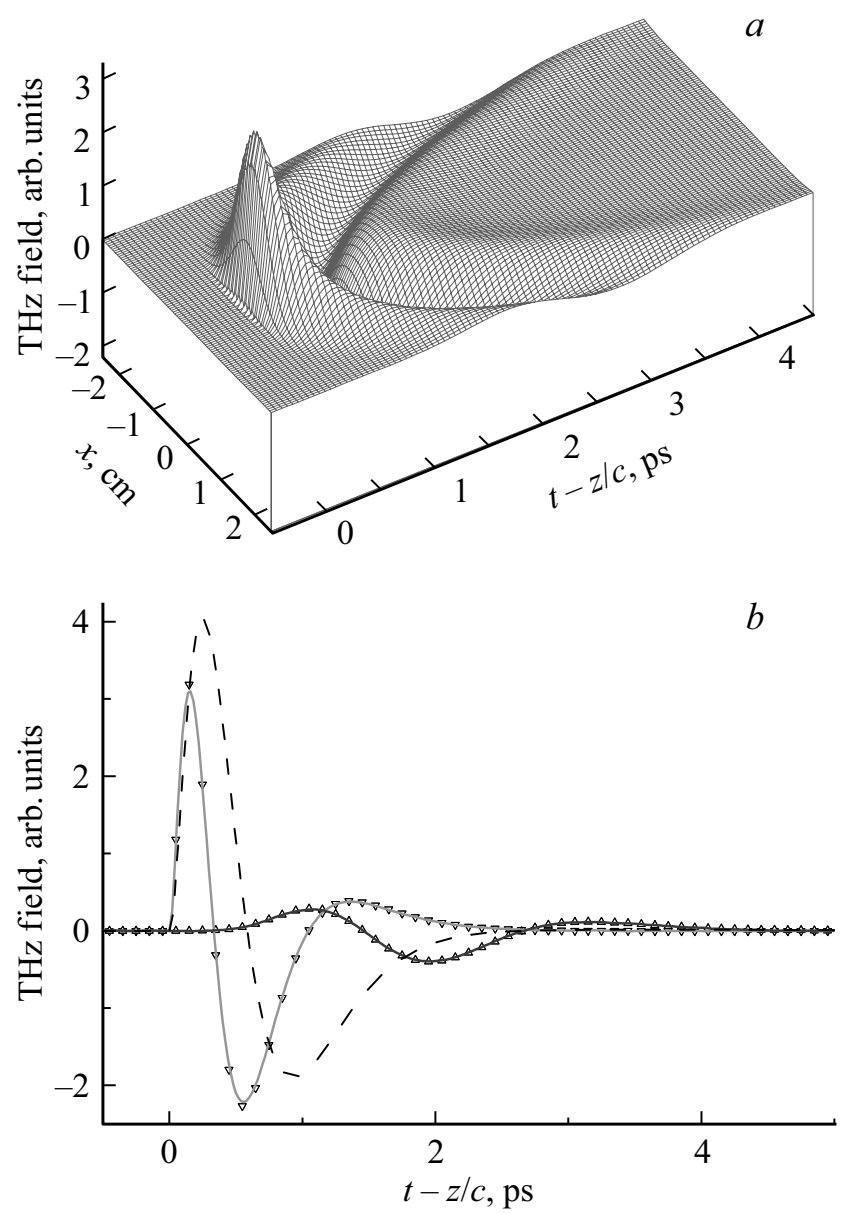

Рис. 2. Пространственно-временное распределение поля ТГц импульса в плоскости $z=50 \mathrm{~cm}$, рассчитанное методом Монте-Карло $(a)$. Временные профили ТГц импульса для двух значений поперечной координаты $x=0$ (1), $2 \mathrm{~cm}$ (2). Сплошные кривые - результат моделирования Монте-Карло, треугольники - результат расчета по формуле (6). Штриховая кривая - начальный профиль ТГц импульса при $z=0$. 


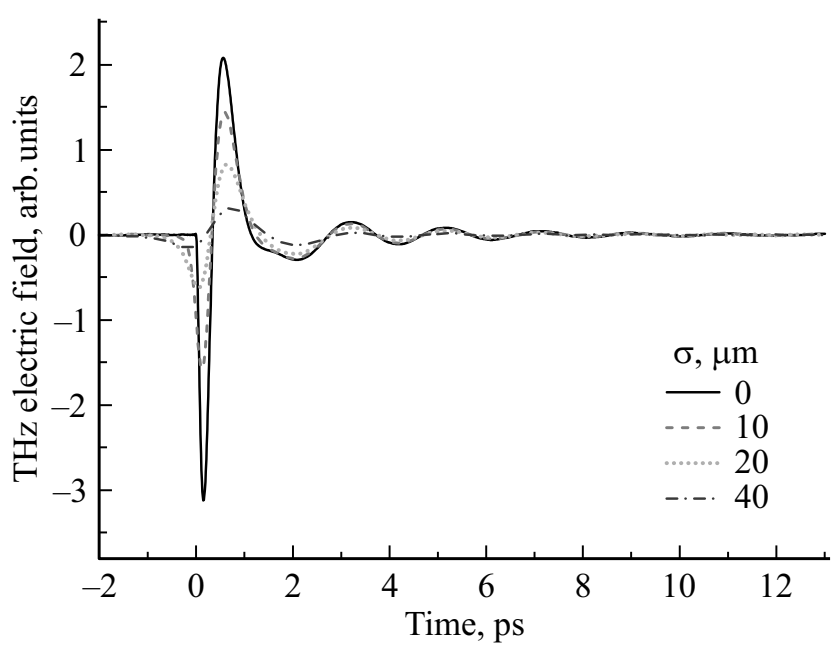

Рис. 3. Рассчитанные методом Монте-Карло временные зависимости когерентного электрического поля ТГц импульса, отраженного от поверхности шероховатой среды, характеризующейся лоренцевым типом дисперсии. $z=50 \mathrm{~cm}$.

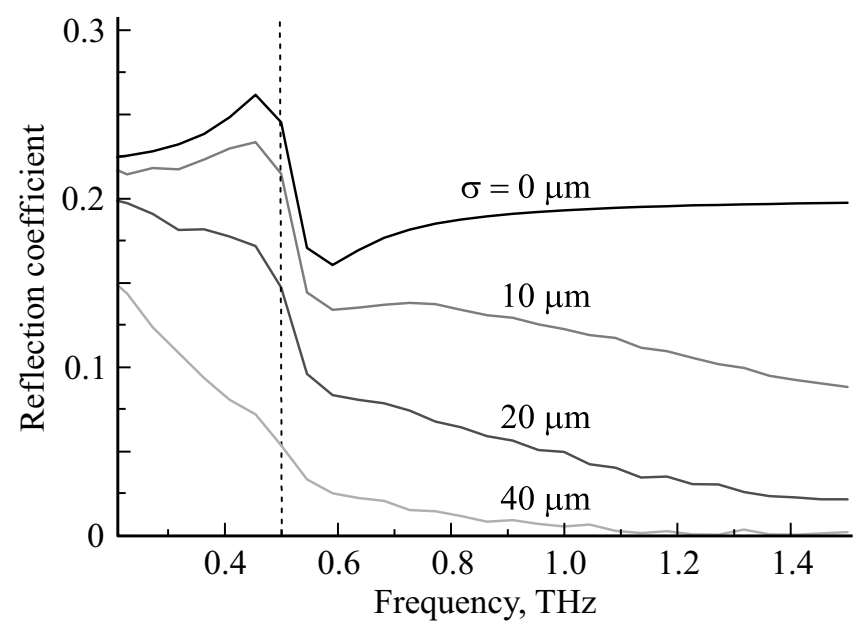

Рис. 4. Спектральные зависимости коэффициента зеркального отражения от среды с шероховатой поверхностью и лоренцевым типом дисперсии. $z=50 \mathrm{~cm}$.

мостью лоренцева типа:

$$
\varepsilon(\omega)=\varepsilon_{\infty}+\frac{\left(\varepsilon_{0}-\varepsilon_{\infty}\right) \omega_{0}^{2}}{\omega_{0}^{2}-\omega^{2}-i \gamma \omega} .
$$

Здесь $\varepsilon_{0}$ и $\varepsilon_{\infty}-$ статическая и высокочастотная диэлектрические проницаемости, $\omega_{0}-$ резонансная частота, $\gamma$ - параметр, характеризующий ширину контура поглощения. Использовались следующие значения параметров: $\omega_{0}=0.5 \mathrm{THz}, \gamma=0.1 \mathrm{THz}, \varepsilon_{\infty}=2.25, \varepsilon_{0}=2.45$, $l=0.2 \mathrm{~cm}, w=0.5 \mathrm{~cm}, \tau=0.2 \mathrm{ps}$.

На рис. 3 представлены временные формы ТГц импульсов, отраженных в зеркальном направлении от шероховатой поверхности дисперсионной среды, рассчитанные для разных значений среднеквадратичной высоты неровностей. Временная зависимость ТГц поля отра- женного импульса демонстрирует наличие осцилляций на резонансной частоте, затухающих с характерным временем затухания $\gamma^{-1}$. Из временных зависимостей рассчитывался амплитудный спектр поля отраженного ТГц импульса. На рис. 4 приведены спектры эффективного коэффициента отражения для среды с дисперсионным законом (7) и шероховатой поверхностью. Этот коэффициент рассчитывался как отношение амплитудного спектра ТГц импульса, отраженного от среды, к амплитудному спектру опорного сигнала, который представлял собой ТГц импульс от гладкой, идеально отражающей поверхности. На рис. 5 этот спектр вычислен по аналитической формуле с использованием фактора $\exp \left(-2 \sigma^{2} \omega^{2} / c^{2}\right)$ для поверхности с нормальным распределением ее возвышений. Видно, что результаты, приведенные на этих рисунках, качественно согласуются и что характерная особенность в спектре отражения на частоте 0.5 ТГц (соответствует длине волны 600 мкм) хорошо различима для поверхностей с характерной высотой неровностей < 40 мкм. При больших значениях высоты неровностей характерный перегиб вблизи резонансной частоты становится практически незаметным. Формальный критерий „исчезновения“ спектральной линии можно ввести требованием слияния соответствующих минимума и максимума в спектре коэффициента отражения, такой критерий приводит к близким критическим значениям сечения рассеяния. Некоторое различие рис. 4 и 5 вызвано тем, что для первого из этих рисунков источник и детектор располагались на конечном расстоянии от шероховатой поверхности, а для второго - полагалось, что это расстояние отвечает дальней зоне применительно к существенному диапазону частот излучения.

Таким образом, из расчетов следует, что рассеяние на шероховатой поверхности с характерной высотой

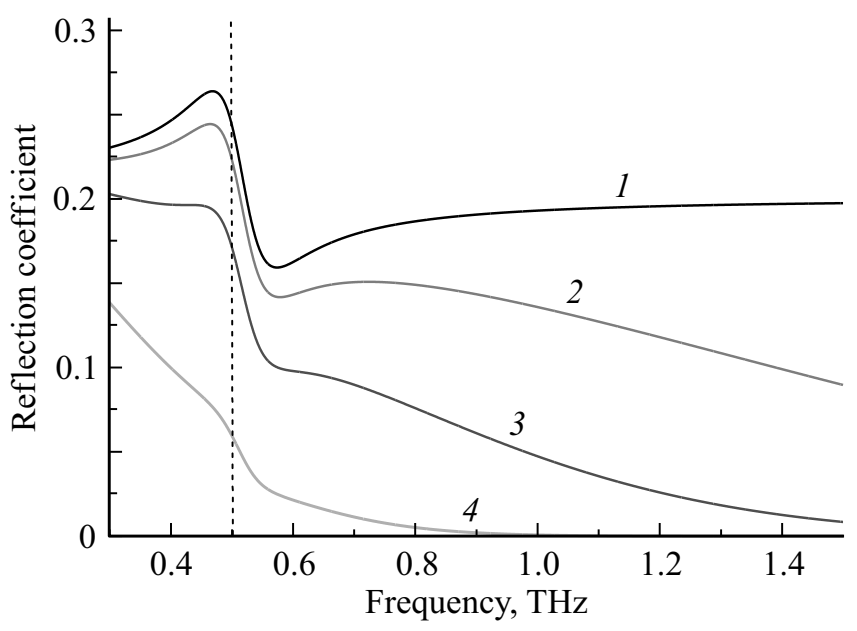

Рис. 5. Аналитические частотные зависимости коэффициента зеркального отражения от среды с лоренцевой дисперсией с различной степенью шероховатости. От линии 1 к линии 4 параметр статистической дисперсии $\sigma^{2}$ возрастает в соответствии с указанными на рис. 4 значениями. 
неровностей, много меньшей длины волны, искажает спектр детектируемого ТГц поля и существенно влияет на регистрацию спектральной особенности. Здесь важно отметить, что вклад в искажение спектра ТГц импульса, отраженного от шероховатой поверхности, дают два эффекта - рассеяние и дифракция (с учетом конечности расположения излучателя и детектора относительно поверхности раздела). Также эти результаты (рис. 5) показывают возможность, во всяком случае качественно, использовать аналитическое приближение для восстановления параметров среды. Действительно, влияние шероховатости границы раздела сред с хорошей степенью точности устраняется домножением измеренного спектра коэффициента отражения на $\exp \left(2 \sigma^{2} \omega^{2} / c^{2}\right)$. Отметим, что совершенно аналогичное аналитическое выражение можно получить и для коэффициента пропускания $[19,20]$, а также для слоистых сред с шероховатыми границами раздела слоев и объемным рассеянием. Практически для этого спектр коэффициента отражения следует умножить на фактор $\exp \left(2 \sigma_{\mathrm{ef}}^{2} \omega^{2} / c^{2}\right)$, в котором эффективное сечение рассеяния $\sigma_{\mathrm{ef}}$ можно подобрать из условия проявления наиболее резких спектральных контуров. Тогда извлечение параметров сред сведется к задаче, рассматриваемой в [21], но с учетом шероховатости границ и случайного объемного рассеяния. Кроме того, если с помощью временного окна разделить сигналы, отраженные различными границами раздела, то спектры отражения от них будут независимы друг от друга. Тогда с помощью подбора значения эффективной дисперсии возвышений для границ слоев и, вообще говоря, сечений объемного рассеяния можно, по крайней мере, приближенно исключить влияние шероховатости и рассеяния на коэффициент отражения от поверхности и от слоистой структуры, не прибегая к сложной процедуре решения обратной задачи, как, например, в [22,23]. При этом для повышения точности необходимо использовать как можно более короткие импульсы диагностирующего излучения, вплоть до субцикловых, методы генерации которых рассматривались, в частности, в работах [24-26].

\section{Заключение}

На основе модифицированного метода Монте-Карло, учитывающего фазу излучения, разработаны алгоритм и программа численного моделирования рассеяния широкополосных импульсов ТГц излучения с неоднородным поперечным профилем на шероховатой поверхности. Рассчитано когерентное поле ТГц импульса с гауссовым поперечным профилем, отраженного от шероховатой поверхности среды с лоренцевым контуром поглощения. Показано, что метод когерентной отражательной ТГц спектроскопии с разрешением во времени оказывается весьма чувствительным к степени шероховатости поверхности исследуемого объекта. Предлагается простой способ исключения или ослабления влияния рассеяния на восстановление характеристик сред в методе импульсной терагерцовой спектроскопии.

Работа поддержана грантами РФФИ 16-52-00109 Бел_а и 16-02-00762, а также грантом БРФФИ № Ф16Р137.

\section{Список литературы}

[1] Zhang $X .-C$., $X u$ J. Introduction to THz Wave Photonics. Springer, 2010. 246 р. Перевод: Си-Чен Чжсан, Джингджю Шю. Терагерцовая фотоника, М.,Ижевск: ИКИ, 2016. $334 \mathrm{c}$.

[2] Terahertz Spectroscopy and Imaging / Ed. by Peiponen K.-E., Zeitler J.A., Kuwata-Gonokami M. Springer Series in Optical Sciences. V. 171. Springer, 2013. 641 p.

[3] Dikmelik Y., Spicer J.B., Fitch M.J., Osiander R. // Opt. Lett. 2006. V. 31. N 24. P. 3653.

[4] Ortolani M., Lee J.S., Schade U., Hubers H.W. // Appl. Phys. Lett. 2008. V. 93. N 8. P. 081906.

[5] Liu H.-B., Chen Y., Bastiaans G.J., Zhang X.-C. // Opt. Expr. 2006. V. 14. N 1. P. 415.

[6] Zurk L.M, Orlowski B., Winebrenner D.P., Thorsos E.I., Leahy-Hoppa M.R., Hayden L.M. // J. Opt. Soc. Am. B. 2007. V. 24. N 9. P. 2238.

[7] Schecklman S., Zurk L.M., Henry S., Kniffin G.P. // J. Appl. Phys. 2011. V. 109. N 9. P. 094902.

[8] Piesiewicz R., Jansen C., Mittleman D., Kleine-Ostmann T., Koch M., Kürner T. // IEEE Trans. Antenn. Propag. 2007. V. 5. N 11. P. 3002.

[9] Théberge F., Châteauneuf M., Dubois J., Désilets S., Lussier L.-S. // Opt. Expr. 2009. V. 17. N 13. P. 10841-10848.

[10] Розанов Н.Н., Сочилин Г.Б., Федоров С.В., Шацев А.Н., Малевич В.Л., Синицын Г.В. // Опт. и спектр. 2017. Т. 123. № 1. C. 137-145. Rosanov N.N., Sochilin G.B., Fedorov S.V., Shatsev A.N., Malevich V.L., Sinitsyn G.V. // Opt. Spectr. 2017. V. 123. N 1. P. 150-157.

[11] Daria V.R., Saloma C., Kawata S. // Appl. Opt. 2000. V. 39. N 28. P. 5244.

[12] $Н$ и M. // Opt. Expr. 2004. V. 12. N 26. P. 6530.

[13] Sawicki J., Kastor N., Xu M. // Opt. Expr. 2008. V. 16. N 8. P. 5728.

[14] Hayakawa C.K., Venugopalan V., Krishnamachari V.V., Potma E.O. // Phys. Rev. Lett. 2009. V. 103. N 4. P. 043903.

[15] Рытов С.М., Кравиов Ю.А., Татарский В.И. Введение в статистическую радиофизику. Ч. ІІ. Случайные поля. М.: Наука, Физматлит, 1978. 465 с. Rytov S.M., Kravtsov Yu.A., Tatarskii V.I. Principles of Statistical Radiophysics 4. Springer, 1989.

[16] Басс Ф.Г., Фукс И.М. Рассеяние волн на статистически неровной поверхности. М.: Наука, Физматлит, 1972. 424 с. Перевод: Bass F.G., Fuks I.M. Wave scattering from statistically rough surfaces. Pergamon Press, 1979.

[17] Ахманов С.A., Выслоух В.А., Чиркин А.С. Оптика фемтосекундных лазерных импульсов. М.: Наука, Физматлит, 1988. 312 c. Akhmanov S.A., Vysloukh V.A., Chirkin A.S. Optics of Femtosecond Laser Pulses. N.Y.: American Institute of Physics, 1992. 
[18] Розанов Н.Н., Сочилин Г.Б., Федоров С.В., Шацев А.Н., Малевич В.Л., Синицын Г.В. // Опт. и спектр. 2017. Т. 123. № 1. C. 132-136. Rosanov N.N., Sochilin G.B., Fedorov S.V., Shatsev A.N., Malevich V.L., Sinitsyn G.V. // Opt. Spectrosc. 2017. V. 123. N 1. P. 146-149.

[19] Ishimaru A. Wave propagation and scattering of random media. V. 2. Multiple scattering, turbulence, rough surfaces and remote sensing. N. Y.; San Francisco; London: Academic Press, 1978. Перевод: Исимару А. Распространение и рассеяние волн в случайно-неоднородных средах. Т. 2. Многократное рассеяние, турбулентность, шероховатые поверхности и дистанционное зондирование. М.: Мир, $1981.315 \mathrm{c}$.

[20] Mayank Kaushik, Brian W.-H. Ng, Bernd M. Fischer, Derek Abbott // Appl. Phys. Lett. 2012. V. 100. P. 241110.

[21] Born M., Wolf E. Principles of optics. Pergamon Press, 1964. Перевод: Борн М., Воль $\varnothing$ Э. Основы оптики. М.: Наука, 1970. $856 \mathrm{c}$.

[22] Jose A. Hejase, Edward J. Rothwell, Premjeet Chahal // IEEE Trans. Terahertz Sci. Technol. 2013. V. 3. N 5. P. 656-665.

[23] Зайщев К.И., Гавдуи А.А., Лебедев С.П., Карасик В.Е., Юрченко С.О. // Опт. и спектр. 2015. Т. 118. № 4. C. 582-593. Zaytsev K.I., Gavdush A.A., Lebedev S.P., Karasik V.E., Yurchenko S.O. // Opt. Spectrosc. 2015. V. 118. N 4. P. $552-562$.

[24] Архипов Р.М., Пахомов А.В., Архипов М.В., Бабушкин И., Толмачев Ю.А., Розанов Н.Н. // Письма в ЖЭТФ. 2017. Т. 105. № $6 . \quad$ С. 388-400. Arkhipov R.M., Pakhomov A.V., Arkhipov M.V., Babushkin I., Tolmachev Yu.A., Rosanov N.N. // JETP Letters. 2017. V. 105. N 6. P. $408-418$.

[25] Архипов Р.М., Архипов М.В., Пахомов А.В., Бабушкин И., Розанов Н.Н. // Опт. и спектр. 2017. Т. 122. № 6. C. 993-999. Arkhipov R.M., Arkhipov M.V., Pakhomov A.V., Babushkin I., Rosanov N.N. // Opt. Spectr. 2017. V. 122. N 6. P. 949-954.

[26] Пахомов А.В., Архипов Р.М., Архипов М.В., Бабушкин И., Розанов Н.Н. // Опт. и спектр. 2017. Т. 123. № 6. C. 901-909. Pakhomov A.V., Arkhipov R.M., Arkhipov M.V., Babushkin I., Rosanov N.N. // Opt. Spectr. 2017. V. 123. N 6. P. 913-917. 